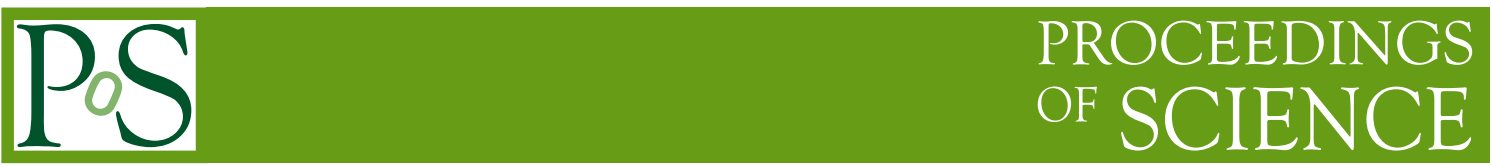

\title{
Neutrino decoherence due to radiative decay
}

\author{
Konstantin Stankevich* \\ Department of Theoretical Physics, Moscow State University, 119992 Moscow, Russia \\ E-mail:kl.stankevich@physics.msu.ru
}

\author{
Alexander Studenikin \\ Department of Theoretical Physics, Faculty of Physics, Lomonosov Moscow State University, \\ Moscow 119991, Russia \\ Joint Institute for Nuclear Research, Dubna 141980, Moscow Region, Russia \\ E-mail: studenikesrd.sinp.msu.ru
}

\begin{abstract}
The phenomena of neutrino oscillations can proceed only in the case of the coherent superposition of neutrino mass states. An external environment can modify a neutrino evolution in a way that conditions for the coherent superposition of neutrino mass states are violated. Such a violation results in quantum decoherence of neutrino states and leads to suppression of flavor neutrino oscillations. We consider the influence of the neutrino radioactive decay in dense media and an external electromagnetic field on neutrino oscillations, and the corresponding damping of neutrino oscillations in the presence of an electron media and electromagnetic field is calculated. The formalism of quantum electrodynamics of open systems is used in the performed evaluations. The studied phenomena can be significant for description of neutrino oscillations in extreme conditions of astrophysical environments peculiar to supernovae, neutron stars or quasars.
\end{abstract}

The 39th International Conference on High Energy Physics (ICHEP2018)

4-11 July, 2018

Seoul, Korea

${ }^{*}$ Speaker. 
We continue our studies [1] of neutrino quantum decoherence in extreme environments. Now consider the influence of the neutrino radioactive decay in dense media on neutrino oscillations. We consider the influence of the neutrino radioactive decay in dense media and an external electromagnetic field on neutrino oscillations, and the corresponding damping of neutrino oscillations in the presence of an electron media and electromagnetic field is calculated. We start with the quantum Liouville's equation for density matrix of a system composed of neutrino and electromagnetic field:

$$
\frac{\partial}{\partial t} \rho=-i \int d^{3} x[H(x), \rho],
$$

where $H(x)=H_{v}(x)+H_{f}(x)+H_{\text {int }}(x)$ is the Hamiltonian density of the system. $H_{v}(x)$ and $H_{f}(x)$ are the Hamiltonian densities of neutrino system and the electromagnetic field respectively, and $H_{\text {int }}(x)$ describes interaction between neutrino and the field

$$
H_{\text {int }}(x)=j_{\alpha}(x) A^{\alpha}(x)
$$

where $A_{\alpha}$ is the electromagnetic field and $j_{\alpha}(x)$ is a current density of neutrino $j_{\alpha}(x)=\overline{v^{\prime}}(x) \Gamma_{\alpha} v(x)$, $v(x)$ is the neutrino field and $\Gamma_{\alpha}=U_{e v}^{*} U_{e v^{\prime}} \tau_{\alpha \beta} \gamma^{\alpha} L$ is an effective electromagnetic vertex

Equation (1) can be formally solved (integrated). Since we are not interested in the evolution of the electromagnetic field its degrees of freedom should be traced out

$$
\rho_{v}\left(t_{f}\right)=\operatorname{tr}_{f}\left(\operatorname{Texp}\left[\int_{t_{i}}^{t_{f}} d^{4} x[H(x), \rho(t)]\right]\right),
$$

where $\rho_{v}(t)=\operatorname{tr}_{f} \rho(t)$ is a density matrix which describes the evolution of a neutrino system. Below we will omit the index " $v$ " in order not to overload formulas. Equation (3) can be reduced to the form of the quantum optical master equation

$$
\begin{aligned}
\frac{\partial}{\partial t} \rho(t)=-i\left[H_{v}, \rho(t)\right]+\kappa\left(\sigma_{-} \rho(t) \sigma_{+}\right. & \left.-\frac{1}{2} \sigma_{+} \sigma_{-} \rho(t)-\frac{1}{2} \rho(t) \sigma_{+} \sigma_{-}\right)+ \\
& +\kappa\left(\sigma_{+} \rho(t) \sigma_{-}-\frac{1}{2} \sigma_{-} \sigma_{+} \rho(t)-\frac{1}{2} \rho(t) \sigma_{-} \sigma_{+}\right)
\end{aligned}
$$

where $H_{v}=\operatorname{diag}\left(\tilde{E}_{1}, \tilde{E}_{2}\right)$ and the decoherence parameter $\kappa=\sin ^{2} 2 \tilde{\theta} \tau^{2} \frac{\Delta}{4 \pi^{2}}(N(2 \Delta)+1)$. The decoherence parameter depends on $\sin ^{2} \tilde{\theta}$ that means that the parameter undergoes the MSW-effect.

The solution of equation (4) gives the probability of neutrino flavour oscillations:

$$
P_{v_{e} \rightarrow v_{\mu}}=\sin ^{2} 2 \tilde{\theta} \sin ^{2}(\Delta t) e^{-\kappa t / 2}+\frac{1}{2}\left(1-\sin ^{2} 2 \tilde{\theta} e^{-\kappa t / 2}-\cos ^{2} 2 \tilde{\theta} e^{-\kappa t}\right) .
$$

This work is supported by the Russian Basic Research Foundation grants No. 16-02-01023 and 17-52-53-133.

\section{References}

[1] K. Stankevich, A. Studenikin "Neutrino quantum decoherence due to entanglement with a magnetic field” PoS EPS-HEP2017 (2018) 645 arXiv:1801.08896 